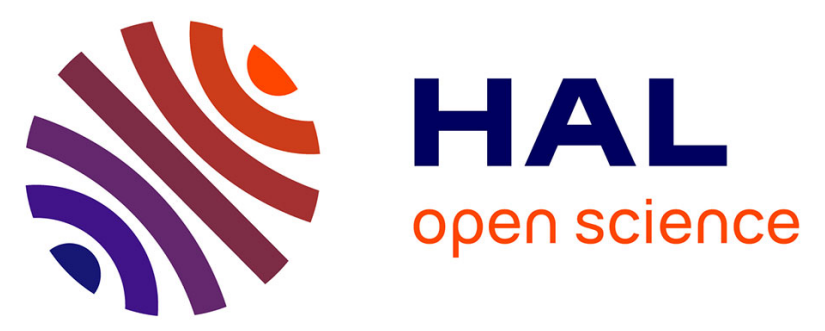

\title{
Electronic Structure Sensitivity to Surface Disorder and Nanometer-Scale Impurity of 2D Titanium Carbide MXene Sheets as Revealed by Electron Energy-Loss Spectroscopy
}

T. Bilyk, M. Benchakar, M. Bugnet, L. Loupias, P. Chartier, H. Pazniak, M.-L. David, A. Habrioux, S. Celerier, J. Pacaud, et al.

\section{To cite this version:}

T. Bilyk, M. Benchakar, M. Bugnet, L. Loupias, P. Chartier, et al.. Electronic Structure Sensitivity to Surface Disorder and Nanometer-Scale Impurity of 2D Titanium Carbide MXene Sheets as Revealed by Electron Energy-Loss Spectroscopy. Journal of Physical Chemistry C, 2020, 124 (49), pp.27071-27081. 10.1021/acs.jpcc.0c06798 . hal-03103681

\section{HAL Id: hal-03103681 \\ https://hal.science/hal-03103681}

Submitted on 8 Jan 2021

HAL is a multi-disciplinary open access archive for the deposit and dissemination of scientific research documents, whether they are published or not. The documents may come from teaching and research institutions in France or abroad, or from public or private research centers.
L'archive ouverte pluridisciplinaire HAL, est destinée au dépôt et à la diffusion de documents scientifiques de niveau recherche, publiés ou non, émanant des établissements d'enseignement et de recherche français ou étrangers, des laboratoires publics ou privés. 


\title{
Electronic Structure Sensitivity to Surface
}

Disorder and Nanometer Scale Impurity of 2D

Titanium Carbide MXene Sheets as Revealed

\section{by Electron Energy-Loss Spectroscopy}

T. Bilyk, ${ }^{*}{ }^{\dagger}$ M. Benchakar, ${ }^{\ddagger}$ M. Bugnet,,${ }^{\llbracket}$ L. Loupias, ${ }^{\ddagger}$ P. Chartier, ${ }^{\dagger}$ H. Pazniak, ${ }^{\dagger}$ M.-L. David ${ }^{\dagger}$ A. Habrioux, ${ }^{\ddagger}$ S. Celerier, ${ }^{\ddagger}$ J. Pacaud, ${ }^{\dagger}$ and V. Mauchamp ${ }^{*, \dagger}$

†Institut Pprime - UPR 3346 - CNRS, Université de Poitiers, ISAE-ENSMA, BP 30179, 86962 Futuroscope-Chasseneuil Cedex, France

$\ddagger$ Institut de Chimie des Milieux et Matériaux de Poitiers (IC2MP), Université de Poitiers, CNRS, F-86073 Poitiers, France

๑Université de Lyon, INSA Lyon, UCBL Lyon 1, MATEIS - UMR 5510 CNRS, 7 av. Jean Capelle, F-69621 Villeurbanne Cedex, France

E-mail: thomas.bilyk@univ-poitiers.fr; vincent.mauchamp@univ-poitiers.fr

\begin{abstract}
Two-dimensional (2D) carbides and/or nitrides (so-called MXenes) are among the latest and largest family of 2D materials. Due to their 2D nature and their unique properties of hydrophilicity, good metallic conductivity and structural diversity, these materials are intensively studied for sensing applications or as supports for nanomaterials toward, e.g., plasmonics, catalytic or energy storage applications. For these potential usages, the extent to which the electronic properties of MXene sheets are modified
\end{abstract}


upon functionalization or intercalation is critical, and an optimized non-destructive probing of the interaction between MXene layers and functionalization is important to be determined. Here, these issues are addressed using a combination of first principle simulations and electron energy loss spectroscopy (EELS) experiments performed at the nanoscale on $\mathrm{Ti}_{3} \mathrm{C}_{2} \mathrm{~T}_{x}$ and $\mathrm{Ti}_{2} \mathrm{CT}_{x}$ MXene multilayers, where $\mathrm{T}$ are the surface functionalization groups. Based on a detailed analysis of the carbon and surface groups $\mathrm{K}$ edge fine structure, we show that the $\mathrm{C}-\mathrm{K}$ edge is an ideal marker for surface induced electronic structure modifications in the $\mathrm{Ti}_{n+1} \mathrm{C}_{n}$ conducting core. These results highlight how a nanometer scale impurity can very locally interact with a $\mathrm{Ti}_{3} \mathrm{C}_{2} \mathrm{~T}_{x}$ multilayer and modify its electronic structure. This approach allows to discriminate between surface and core alteration of the $\mathrm{Ti}_{3} \mathrm{C}_{2} \mathrm{~T}_{x}$ layers. Finally, the higher sensitivity to surface states of the $\mathrm{Ti}_{n+1} \mathrm{C}_{n}$ conducting core in $\mathrm{Ti}_{2} \mathrm{CT}_{x}$ as compared to $\mathrm{Ti}_{3} \mathrm{C}_{2} \mathrm{~T}_{x}$ is discussed. We expect these results to offer an approach for understanding MXenes' behavior, and especially characterize their interactions with other nanomaterials when used in composites.

\section{Introduction}

In the growing world of 2D materials, transition metal carbides and/or nitrides - so-called MXenes (M standing for a transition metal and X standing for $\mathrm{C}$ and/or $\mathrm{N}$ ) - have raised considerable interest in the scientific community since their discovery in $2011 .^{1,2}$ This large family of $2 \mathrm{D}$ systems occupies a special place in materials science for at least two reasons. Firstly, these materials combine high conductivity and hydrophilicity, ${ }^{3,4}$ with utmost benefits for many applications involving aqueous media, ${ }^{5,6}$ requiring good electronic properties, ${ }^{7-10}$ or easy processing. ${ }^{11}$ Secondly, MXenes are obtained from the exfoliation of the A-element layers (mainly from columns 13 and 14 of the periodic table) from the so-called MAX phases ${ }^{12}$ a 150-plus member family of layered ternary carbides or nitrides with chemical composition $\mathrm{M}_{n+1} \mathrm{AX}_{n}(\mathrm{n}=1-3)-{ }^{13}$ thus offering considerable opportunities in terms of chemical compo- 
sition modulations. In addition, depending on the value of $n$ in the parent MAX phase, the corresponding $\mathrm{M}_{n+1} \mathrm{X}_{n}$ single sheet thickness can be adjusted from three to seven atomic layers, giving direct opportunities to probe the structure/chemistry/properties relationship in this class of materials. As such, by changing the MAX phase precursor composition (i.e. the $\mathrm{M}$ or $\mathrm{X}$ elements) or structure (i.e. the value of $n$ ), one can obtain a large number of different MXenes with markedly different physico-chemical properties. ${ }^{14-16}$

Another key feature in designing the MXene properties is the possibility to modify their surface functionalization groups $\mathrm{T}$ (i.e. $\mathrm{T}=-\mathrm{F},-\mathrm{OH},-\mathrm{O}$ or $-\mathrm{Cl}$ ), which are usually inherited from the exfoliation step of the $\mathrm{M}_{n+1} \mathrm{AX}_{n}$ phases, and lead to the following formula for the MXenes: $\mathrm{M}_{n+1} \mathrm{X}_{n} \mathrm{~T}_{x}(\mathrm{x} \approx 2)$. From the electronic structure point of view, these surface groups being directly bounded to the transition metal atomic layer, they modify the M $d$ band occupation ${ }^{17}$ and induce a dramatic effect on the MXenes' electronic or magnetic properties. ${ }^{18,19}$ The crucial role of the surface groups and the fundamental importance of their chemical nature has been demonstrated recently by showing that fully $\mathrm{Cl}, \mathrm{S}$ or Se terminated $\mathrm{Nb}_{2} \mathrm{C}$ exhibit superconductivity bewteen 4.5 and $6.4 \mathrm{~K}^{20}$ As a result, a huge interest exists to characterize and control the MXene surface structure/chemistry, ${ }^{21-24}$ either during the etching process using different etching protocols, ${ }^{25-28}$ or via post-treatments in order to control the MXene properties or induce new functionalizations. Understanding how the MXene surface affects the MX conducting core is also extremely important when designing MXene-based composites where MXenes can be used as conducting matrices. ${ }^{29}$ In that respect, the non-selective van der Waals interactions have been shown to play a positive role in the homogenous dispersion of transition metal oxide nanostructures on $\mathrm{Ti}_{3} \mathrm{C}_{2}$ nanosheets, ${ }^{30}$ whereas the strong interaction between a layered cobalt double hydroxide (Co-LDH) and its MXene support has been reported to be key for the electrocatalytic performances of Co-LDH@Ti ${ }_{3} \mathrm{C}_{2}$ composites. $^{31}$

Although of fundamental and practical interest, direct experimental evidences of the role of the MXene surface functionalization on their intrinsic electronic properties are far from 
easy to obtain. One reason is the highly reactive surface of MXenes that lead to undesired modifications in poorly controlled atmospheres. Another reason is the complexity to correlate the changes observed in properties measured on a macroscopic scale (e.g. electrical conductivity) with structural considerations requiring to go down to the single flake or atomic level. In particular Sang et al., ${ }^{32}$ by combining atomically resolved STEM imaging with DFT simulations and conductivity measurements performed at the single flake level, have shown that the density of $\mathrm{Ti}$ vacancies induced by the etching process in $\mathrm{Ti}_{3} \mathrm{C}_{2} \mathrm{~T}_{x}$ layers do not significantly affect their metallic character and that the number of layers in a $\mathrm{Ti}_{3} \mathrm{C}_{2} \mathrm{~T}_{x}$ multilayer has an effect on their conductivity. In this context, transmission electron microscopy (TEM) offers very interesting opportunities as it allows to analyze the MXenes structure in high vacuum, probing surface changes through in situ thermal annealing or electron beam irradiation for instance, ${ }^{33,34}$ while characterizing the induced structural modifications using both imaging and spectroscopy techniques. Specifically, electron energy loss spectroscopy (EELS), which is extremely relevant in the study of light elements, can be used to investigate the T-groups desorption by focusing on the F-K and O-K edges. In addition, the fine structure at these edges reflect the unoccupied electron density of states (mainly $p$ symmetry) projected on the corresponding atoms, and give access to the electronic structure of the material. With these possibilities, the correlation between T-groups desorption and changes in the electronic conductivity, ${ }^{34}$ or plasmonic properties ${ }^{35}$ of MXene flakes have been recently evidenced, in agreement with earlier theoretical predictions. ${ }^{36}$

In this study, we expand the understanding of the role of surface defects (i.e. T-groups vacancies or disorder, adsorbed species) on the electronic structure of Ti-based MXenes using (Scanning)TEM-EELS. This issue, together with the functionalization, is very general to 2D materials. ${ }^{37,38}$ We focus on two benchmark MXenes, namely $\mathrm{Ti}_{3} \mathrm{C}_{2} \mathrm{~T}_{x}$ (the most studied MXene to date $\left.{ }^{14}\right)$ and $\mathrm{Ti}_{2} \mathrm{CT}_{x}$, which differ mainly from their elementary thickness (5 and 3 atomic layers in the MX core respectively). By revealing the information contained in the T-K or C-K edge fine structure using first-principles simulations (density functional theory 
$(\mathrm{DFT})$ ), we obtain a direct analysis of the way the chemical bonding of these elements is altered when modifying the MXene surface. Here, we show that the $\mathrm{C}-\mathrm{K}$ edge is a very effective probe to investigate surface disorder: it allows separating the role of surface defects (whether intrinsic like T-vacancies or disorder, or extrinsic like adsorbed impurities) from core defects on the electronic structure of the material. In particular, we emphasize how the $\mathrm{C}-\mathrm{K}$ edge can be used to define the interaction between adsorbed species or nanomaterials and a $\mathrm{Ti}_{3} \mathrm{C}_{2} \mathrm{~T}_{x}$ support, which is a result of utmost relevance in the study of MXene-based systems, ${ }^{31}$ and to determine the defects induced by different etching environments on the MX core. Finally, we discuss the higher sensitivity to defects of the $\mathrm{Ti}_{n+1} \mathrm{C}_{n}$ conducting core in $\mathrm{Ti}_{2} \mathrm{CT}_{x}$ as compared to $\mathrm{Ti}_{3} \mathrm{C}_{2} \mathrm{~T}_{x}$, with possible important repercussions for sensing applications.

\section{METHODS}

DFT simulations Simulations of C-K, O-K and F-K edges were performed using density functional theory (DFT) calculations. The WIEN2k code, based on the full-potential (linearized) augmented plane-wave plus local orbitals method, was used. ${ }^{40,41}$ Calculations were performed in the generalized gradient approximation using the GGA-PBE exchange and correlation functional. ${ }^{42}$ The basis set convergence was achieved using a $\mathrm{R}_{M T} \cdot \mathrm{K}_{\max }$ product of 8, with $\mathrm{R}_{M T}$ the smallest atomic sphere and $\mathrm{K}_{\max }$ the plane wave cut-off. The magnitude of the largest vector in the charge-density Fourier expansion, Gmax was set to 14 . The amount of k-points in the first Brillouin zone was set to 1500 for all cases, leading to 100-150 k-points in the irreducible Brillouin zone depending on the crystal cell. These three parameters were well converged. In particular, for the considered experimental broadening of $\sim 1$ $\mathrm{eV}$, more stringent convergence threshold values lead to identical spectra. The sizes of the

Muffin-Tin (atomic sphere) radii were changed from one termination to another depending on the interatomic distances; these sizes are given in Table 1. The $\mathrm{Ti}_{3} \mathrm{C}_{2} \mathrm{~T}_{2}$ MXene struc- 
ture was described in the $\mathrm{P}_{3} / \mathrm{mmc}$ space group (similar to the parent MAX phase) for all calculations with the following unit-cell parameters (determined from a previous study): ${ }^{43}$ $\mathrm{a}=\mathrm{b}=3.058 \AA$ and $\mathrm{c}=19.59 \AA$. The Wyckoff positions of the atoms for $\mathrm{Ti}_{3} \mathrm{C}_{2} \mathrm{~F}_{2}$ were: one titanium atom in the 2 a position $(0 ; 0 ; 0)$; one titanium atom in the $4 \mathrm{f}$ position $\left(\frac{2}{3} ; \frac{1}{3} ; \mathrm{z}_{\text {Tis }}\right)$, with $\mathrm{z}_{T i s}=0.119727$; one carbon atom in the $4 \mathrm{f}$ position $\left(\frac{2}{3} ; \frac{1}{3} ; \mathrm{z}_{C}\right)$, with $\mathrm{z}_{C}=0.565283$; and one fluorine atom in the $4 \mathrm{e}$ Wyckoff position $\left(0 ; 0 ; \mathrm{z}_{F}\right)$, with $\mathrm{z}_{F}=0.183167$. When the surface terminations were changed to oxygen or vacancies, a relaxation of the atomic positions was done using WIEN2k keeping the space group and the cell parameters constant. For the $\mathrm{Ti}_{2} \mathrm{CT}_{2}$ MXenes, the structures given by Xie et al. ${ }^{44}$ were used. The MXenes are single layers separated by $20 \AA$ of vacuum. The Muffin-Tin radii are also given in table 1 .

Table 1: Atomic sphere radii for all elements in the different simulations (given in bohr units).

\begin{tabular}{c||cccc} 
& $R_{M T}^{T i}$ & $R_{M T}^{C}$ & $R_{M T}^{F}$ & $R_{M T}^{O}$ \\
\hline \hline $\mathrm{Ti}_{3} \mathrm{C}_{2} \mathrm{~F}_{2}$ & 2.08 & 1.74 & 1.98 & \\
$\mathrm{Ti}_{3} \mathrm{C}_{2} \mathrm{O}_{2}$ & 1.95 & 1.83 & & 1.77 \\
$\mathrm{Ti}_{3} \mathrm{C}_{2} \mathrm{~F}_{1} \mathrm{O}_{1}$ & 1.95 & 1.76 & 1.94 & 1.77 \\
$\mathrm{Ti}_{2} \mathrm{CF}_{2}$ & 2.06 & 1.75 & 1.96 & \\
$\mathrm{Ti}_{2} \mathrm{CO}_{2}$ & 1.95 & 1.85 & & 1.77 \\
$\mathrm{Ti}_{2} \mathrm{C}$ & 2.17 & 1.77 & &
\end{tabular}

Concerning the description of mixed surface terminations, the $\mathrm{Ti}_{3} \mathrm{C}_{2} \mathrm{~T}_{x}$ system was either described using the crystal structures described before when the virtual crystal approximation was used, or considering $2 \times 2 \times 1$ supercells (the supercells were also used when investigating the role of vacancies). For the supercells, and in order to save computational time, the VASP ${ }^{45,46}$ software based on the projector augmented wave method was used for structural relaxations, keeping the unit cell parameters constant, with forces converged below $0.01 \mathrm{eV} / \AA$. The PAW atomic configurations were $2 \mathrm{~s}^{2} 2 \mathrm{p}^{2}$ for $\mathrm{C}, 2 \mathrm{~s}^{2} 2 \mathrm{p}^{5}$ for $\mathrm{F}, 2 \mathrm{~s}^{2} 2 \mathrm{p}^{4}$ for $\mathrm{O}$ and $3 s^{2} 3 \mathrm{p}^{6} 3 \mathrm{~d}^{2} 4 \mathrm{~s}^{2}$ for Ti. The energy cut-off restricting the plane wave basis set was converged to $700 \mathrm{eV}$. A $22 \times 22 \times 3 \mathrm{k}$-point mesh was used to sample the first Brillouin zone using a Monkhorst-Pack grid. The atom equilibrium positions were checked regarding forces 
after SCF calculation using WIEN2k.

The TELNES program ${ }^{47}$ implemented in WIEN2k was used to simulate the EEL spectra. The program allows calculating the double differential inelastic scattering cross section in a fully relativistic approach taking into account the different experimental parameters (e.g., zone axis, collection angle, acceleration voltage). Unless specified otherwise, the simulations of the $\mathrm{K}$ edges of the different elements were performed in the final state approximation, i.e. considering a core hole on the excited atom. To avoid the interaction of the core hole with its repeated image due to periodic boundary conditions, $2 \times 2 \times 1$ supercells were also necessary. As the excited atom is treated as an impurity, the resulting space group is P3m1. Local Orbitals with a linearization energy of 2 Ry above the Fermi energy were used in order to get a better description of the inelastic scattering cross section at high energy. The simulations were aligned to the experimental energy scale.

Sample preparation $\mathrm{The} \mathrm{Ti}_{n+1} \mathrm{C}_{n} \mathrm{~T}_{x}$ samples were prepared by etching the aluminum from the $\mathrm{Ti}_{n+1} \mathrm{AlC}_{n}$ MAX phase powders as described in the supporting information-section 1 for the HF-pyridine process and our previous papers. ${ }^{22,26}$ After synthesis, the MXene were dried and stored in glove box under $\mathrm{N}_{2}$ atmosphere. The TEM samples were either prepared by directly collecting the MXene powders on a TEM copper grid covered with lacy carbon films, or by first dispersing the MXenes in pure water before depositing a drop of the suspension on a TEM grid. The $\mathrm{Ti}_{n+1} \mathrm{C}_{n} \mathrm{~T}_{x}$ MXenes are then probed with basal plane perpendicular to the electron beam.

(S)TEM experiments Except for the data of Figure 4, the EEL spectra were recorded in TEM image mode using a JEOL 2200FS transmission electron microscope equipped with an in-column omega filter, operating at $200 \mathrm{kV}$ and using collection semi-angles ranging from 2.5 to $7 \mathrm{mrad}$. The zero-loss peak full width at half maximum was around $1 \mathrm{eV}$. The thickness relative to the inelastic mean free path of the electrons was below 0.3 for the probed MXene multilayers. For core spectra, the beam focalization was adjusted to 
optimize the signal to noise ratio while limiting the irradiation damages. The probed area were approximately 0.01 to $0.1 \mu \mathrm{m}^{2}$. In these conditions, no evolution of the EEL spectra is visible from irradiation induced damage. In order to get spatially resolved information, the STEM-EELS data of Figure 4 were acquired at $300 \mathrm{kV}$ using a FEI Titan ETEM G2 equipped with a GIF-tridiem spectrometer, and achieving a very similar energy resolution of $0.9 \mathrm{eV}$. The spectrum image (SI) was acquired over $130 \mathrm{~nm}, 21 \times 21 \mathrm{px}^{2}$, with a dwell time of $0.5 \mathrm{~s} / \mathrm{px}$. In these conditions, no degradation of the sample was observed during the acquisition of the EEL spectra in STEM mode. All spectra were background subtracted using an inverse power law and deconvolved from multiple scattering when necessary.

\section{Results and discussion}

Although the C-K edge in Al-based MAX phases has been shown to be insensitive to the Al layers, ${ }^{48,49}$ thus giving a direct picture of the electronic structure of the MX layers, the C-K edge in $\mathrm{Ti}_{3} \mathrm{C}_{2} \mathrm{~T}_{x}$ is clearly sensitive to the functionalization of the MXene sheets and, as a consequence, to surface effects. As an illustration, Figure 1-a gives a comparison between different $\mathrm{C}-\mathrm{K}$ edges simulated considering a $\mathrm{Ti}_{3} \mathrm{C}_{2}$ multilayer (ML) in which the MXene sheets are either functionalized with -F, -O (the $\mathrm{T}$ groups being considered in the most stable position, i.e. pointing towards the volume titanium atom $\mathrm{Ti}_{v}$ - see the structural model in Figure 1-a) ${ }^{24,43,44}$ or not functionalized. It clearly attests the strong impact of the nature/presence of the T-groups on the first peak $\mathrm{A}$, and the much smaller effect on the other peaks (labelled B to D). OH terminations were not considered here since it has been shown to give results very similar to $\mathrm{F}$ terminations. ${ }^{22}$ Since it is well known that the interlayer distance in MXenes can significantly vary depending on the nature of $\mathrm{T}$ and inserted

cations, the relative humidity or the drying conditions used after synthesis, ${ }^{4,26}$ equivalent simulations were also performed on single layers in order to consider the two extreme cases in terms of structural description. Although the stacking influences the intensity of peak A 
in $\mathrm{Ti}_{3} \mathrm{C}_{2} \mathrm{~F}_{2}$, it has a smaller effect on $\mathrm{Ti}_{3} \mathrm{C}_{2} \mathrm{O}_{2}$ and no effect on $\mathrm{Ti}_{3} \mathrm{C}_{2}$. As a consequence, any possible difference in interlayer distance arising from stacking effects cancels out when considering a mixture of terminations, a situation much more relevant for the comparison with experimental spectra. This is shown in Figure 1-b where the C-K edges simulated for a carbon atom below a MXene surface functionalized with $25 \% F / 75 \%$ O or above a surface functionalized with $75 \% \mathrm{~F} / 25 \% \mathrm{O}$ are given. These simulations were obtained considering either stacked MXene sheets or single layer: in each case no major difference appears between the two structural models. In addition, these spectra confirm the sensitivity of the C-K edge in the energy range of the peak A (highlighted in grey) to changes in the surface configuration of the MXene layer. Indeed, the simulations for a carbon atom below the $25 \% \mathrm{~F} / 75 \% \mathrm{O}$ surface differs from that obtained above the $75 \% \mathrm{~F} / 25 \% \mathrm{O}$ surface only in the energy range corresponding to this peak.

As mentioned previously, using a single termination is oversimplified in the MXene description and different mixings between the T-groups (considering $x=2$ in the MXene composition, which corresponds to a complete surface saturation) have been considered. The resulting simulations, and corresponding experiment, are given in Figure 1-c where the $\mathrm{F} / \mathrm{O}$ ratio has been changed from 3 to $1 / 3$. The disorder has been simulated considering the virtual crystal approximation (VCA) in which the $\mathrm{O} / \mathrm{F}$ mixing is modeled by considering a virtual $\mathrm{T}$ atom of effective atomic number representing the average $\mathrm{O} / \mathrm{F}$ composition, 8.5 for $50 \% \mathrm{~F} / 50 \% \mathrm{O}$ for instance. The validity of the VCA to simulate the role of surface mixing on the $\mathrm{C}-\mathrm{K}$ edge can be understood from the fact that the T-groups have a very small contribution around the Fermi level and in the unoccupied bands (see the electronic densities of states, DOS, given in supporting information-section 2). Since, the C-K edge is a probe of the projected unoccupied C- $p$ DOS, and since the electronic structure above $\mathrm{E}_{F}$ is dominated by the Ti- $d$ states, the description of the surface disorder in terms of the VCA is reliable to discuss this particular energy range of the electronic structure of the material. As an illustration, the comparison between the total DOS of the $\mathrm{Ti}_{3} \mathrm{C}_{2} \mathrm{OF}$ system as obtained from 

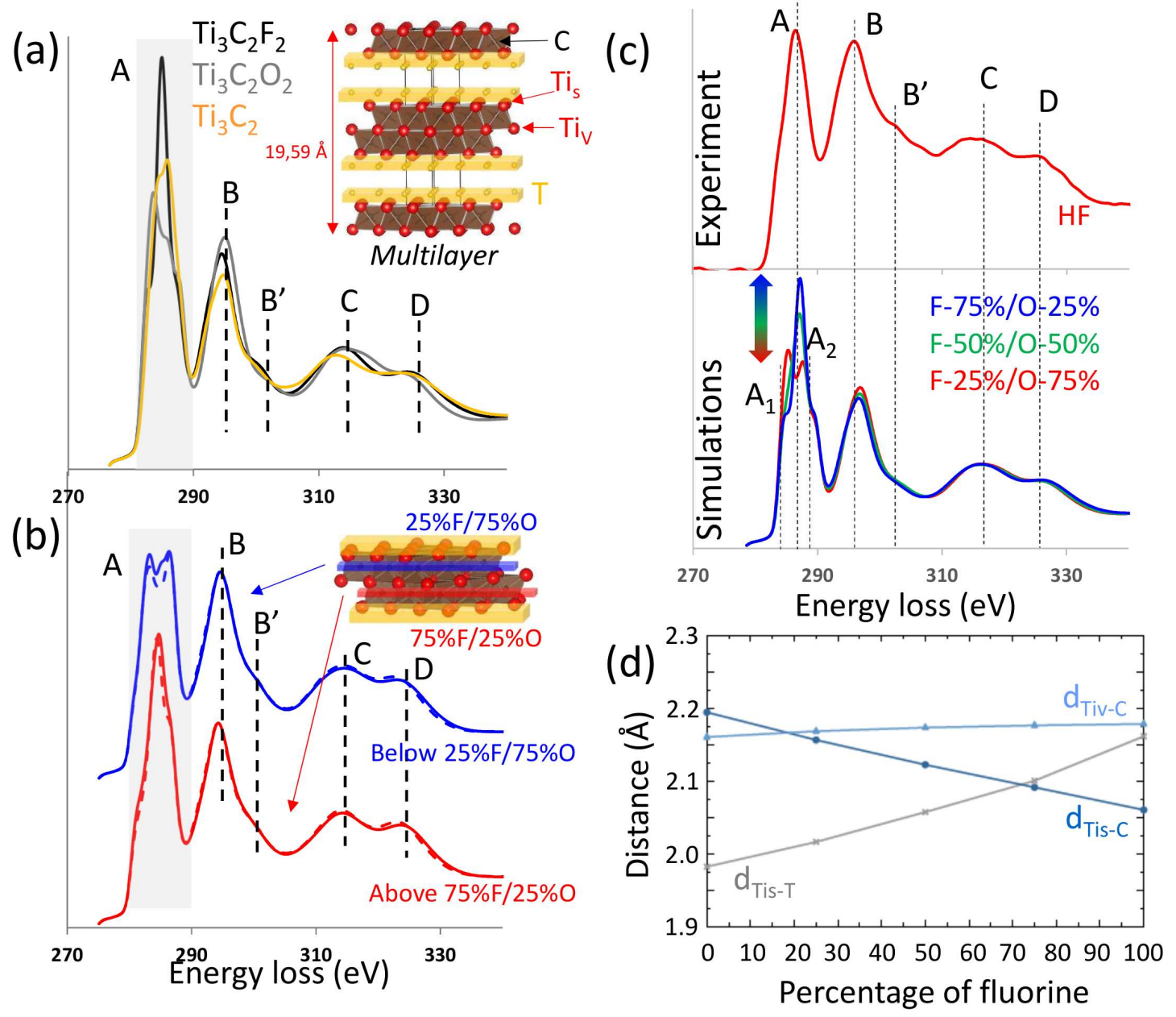

Figure 1: (a) DFT simulations of the C-K edges for different surface terminations: $\mathrm{F}_{2}$ (black), $\mathrm{O}_{2}$ (grey), and without terminations (orange). The structural model corresponds to a unit cell containing two sheets with a unit cell parameter $\mathrm{c}=19.59 \AA$ and described in the $\mathrm{P} 6_{3} /$ mmc space group (see the inset corresponding to a $3 \times 3 \times 1$ supercell). All structural models were drawn using the VESTA software. ${ }^{50}$ (b) Comparison between the C-K edges simulated for a mixture of terminations $(25 \% \mathrm{~F} / 75 \% \mathrm{O}$ or $75 \% \mathrm{~F} / 25 \% \mathrm{O})$ on the two sides of a MXene sheet. Blue: C-K edge for a carbon atom just below the $25 \% \mathrm{~F} / 75 \% \mathrm{O}$ surface in a multilayer (full line) and corresponding calculation for a single layer (dashed line). Red: C-K edge for a carbon atom just above the $75 \% \mathrm{~F} / 25 \% \mathrm{O}$ surface in a multilayer (full line) and corresponding calculation for a single layer (dashed line). In order to save computational time, these calculations were performed without considering a core-hole. (c) Top - Typical experimental C-K edge recorded on a $\mathrm{Ti}_{3} \mathrm{C}_{2} \mathrm{~T}_{x}$ multilayer obtained from HF exfoliation of $\mathrm{Ti}_{3} \mathrm{AlC}_{2}$ powders (see ref. ${ }^{22}$ for the synthesis protocol). Bottom - Evolution of the fine structure at the $\mathrm{C}-\mathrm{K}$ edge for a mixture of $-\mathrm{F}$ and $-\mathrm{O}$ terminations as obtained considering the virtual crystal approximation (VCA). (d) Evolution of the interatomic distances in the MXene sheet determined after structural relaxation in the VCA and corresponding to different $\mathrm{F} / \mathrm{O}$ ratios (given in $\%$ of fluorine). 
the VCA and from a $2 \times 2 \times 1$ supercell where $\mathrm{O}$ and $\mathrm{F}$ atoms are explicitly treated is given in supporting information (see figure S2): it appears clearly that the main difference between these two DOS is at the bottom of the valence band, where the $\mathrm{O}$ and $\mathrm{F}$ states dominate, and that they are very similar above $\mathrm{E}_{F}$. As a consequence, the C-K edges obtained for the $\mathrm{Ti}_{3} \mathrm{C}_{2} \mathrm{OF}$ composition considering either the VCA or a supercell approach are very similar as evidenced in figure S3 of the supporting information, justifying the use of the VCA as a relevant approach in the present study.

Considering a mixture of $\mathrm{O} / \mathrm{F}$ provides a very good description of the experimental spectra given in the upper panel of Figure 1-c. Specifically, these calculations extend our previous conclusions: any intensity change in structure A is a clear marker of surface disorder and gives a direct correlation with induced electronic structure perturbations within the MXcore. In the present case, increasing the O-content on the MXene surface leads to a decrease in the intensity of peak A. In addition, the C-K edge given in the upper panel of figure 1-c is very similar to the one published in ref., ${ }^{22}$ with only a sligth increase in the intensity of peak $\mathrm{A}$ in the present spectrum. We interpret this variation as another evidence of the sensitivity of peak A to inhomogeneities in the surface coverage of different MXene flakes.

The decrease of the calculated intensity for the peak $\mathrm{A}$ in the $\mathrm{Ti}_{3} \mathrm{C}_{2}\left(\mathrm{O}_{x} \mathrm{~F}_{y}\right)$ system can be understood considering the evolution of the interatomic distances in the sheet. The evolution of the $\mathrm{Ti}_{s}-\mathrm{T}$ (where $\mathrm{Ti}_{s}$ are the $\mathrm{Ti}$ atoms on the surface of the layer - see the structural model in Figure 1-a), $\mathrm{Ti}_{s}-\mathrm{C}$ and $\mathrm{Ti}_{v}-\mathrm{C}$ (where $\mathrm{Ti}_{v}$ are the titanium atoms in the center of the sheet) distances is presented in Figure 1-d. From a $100 \%$-O to $100 \%$-F surface composition, these distances evolve as follows: $\mathrm{d}_{\text {Tis-T }}$ increases by $8.3 \%, \mathrm{~d}_{\text {Tis-C }}$ decreases by $6.5 \%$ and $\mathrm{d}_{\text {Tiv-C }}$ changes only by $-0.8 \%$. Oxygen terminations thus tend to pull the $\mathrm{Ti}_{s}$ towards the surface, the bonding between $\mathrm{Ti}_{s}$ and $\mathrm{C}$ is then modified depending on the average surface functionalization. As a consequence of these changes in the $\mathrm{Ti}_{s}-\mathrm{C}$ and $\mathrm{Ti}_{v}-\mathrm{C}$ distances, the hybridizations between these atoms are modified. As an illustration, figure 2-a shows the hybridization schemes between the C- $p$ states (those involved in the C-K edge) 
and the Ti states (predominantly d states), which dominate the electronic structure around $\mathrm{E}_{F}$, in $\mathrm{Ti}_{3} \mathrm{C}_{2} \mathrm{~F}_{2}$ (left) and $\mathrm{Ti}_{3} \mathrm{C}_{2} \mathrm{O}_{2}$ (right) as revealed by the unoccupied DOS up to 20 $\mathrm{eV}$ above $\mathrm{E}_{F}$. This energy range covers the $\mathrm{C}-\mathrm{K}$ edge up to the structure $\mathrm{B}$ identified in figures 1-a and c. For both $\mathrm{Ti}_{3} \mathrm{C}_{2} \mathrm{~F}_{2}$ and $\mathrm{Ti}_{3} \mathrm{C}_{2} \mathrm{O}_{2}$, the C- $p$ unoccupied DOS mimics rather well the simulated EELS spectra in this energy range since one can clearly identify peaks $\mathrm{A}$ and $\mathrm{B}$ as well as the shoulders $\mathrm{A}_{1}$ and $\mathrm{A}_{2}$ of figure 1-c. That the relative intensities are not exactly the same as those obtained in the simulations of the spectra is likely due to the fact that the EELS spectra were obtained considering an energy dependent broadening to mimic excited states lifetime effects, that EELS spectra properly weight the $\mathrm{p}_{x} / \mathrm{p}_{y}$ and $\mathrm{p}_{z}$ contributions to the EELS signal which is not considered in the DOS calculations and, that the core-hole was not considered in the DOS. Inspection of the left part of figure 2-a shows that peak A mainly corresponds to hybridizations between the C- $p$ states and the surface titanium $d$ states in $\mathrm{Ti}_{3} \mathrm{C}_{2} \mathrm{~F}_{2}$. When replacing $\mathrm{F}$ with $\mathrm{O}$, since the $\mathrm{Ti}_{s}-\mathrm{C}$ distance increases whereas the $\mathrm{Ti}_{v}$-C distance is unchanged, the hybridizations of the $\mathrm{C}$ states with the $\mathrm{Ti}_{s}$ states are reduced in favor of the hybridizations with the $\mathrm{Ti}_{v}$ states: the C- $p$ states involved in peak $A$ are thus redistributed towards the energy ranges of the shoulders $A_{1}$ and $A_{2}$, which correspond to local maxima in the $\mathrm{Ti}_{v}$ DOS of $\mathrm{Ti}_{3} \mathrm{C}_{2} \mathrm{O}_{2}$. Consequently, the spectral weight corresponding to peak A is redistributed on a larger energy range and its maximum intensity is reduced in $\mathrm{Ti}_{3} \mathrm{C}_{2} \mathrm{O}_{2}$ as compared to $\mathrm{Ti}_{3} \mathrm{C}_{2} \mathrm{~F}_{2}$. In addition, the intensity of peak $\mathrm{B}$ is slightly increased in $\mathrm{Ti}_{3} \mathrm{C}_{2} \mathrm{O}_{2}$ so that the intensity ratio between peaks $\mathrm{A}$ and $\mathrm{B}$ is close to one in $\mathrm{Ti}_{3} \mathrm{C}_{2} \mathrm{O}_{2}$ whereas it is greater than one in $\mathrm{Ti}_{3} \mathrm{C}_{2} \mathrm{~F}_{2}$, explaining the evolution in the intensity of the simulated EELS spectra in figure 1-c. From this analysis, the relative intensity between peaks A and B is thus characteristic of the hybridizations between the C- $p$ and $\mathrm{Ti}_{s}$ or $\mathrm{Ti}_{v}$ states, these changes being also directly related to modifications in the $\mathrm{Ti}_{s}-\mathrm{T}$ groups bonding.

Since the $\mathrm{Ti}_{s^{-}} d$ states dominate the electronic structure around the Fermi level $\left(\mathrm{E}_{F}\right)$ in $\mathrm{Ti}_{3} \mathrm{C}_{2} \mathrm{~T}_{x}{ }^{43}$ detecting such a modification of the intensity of peak A most probably provides 
an evidence for the modification of the electronic properties of the $\mathrm{Ti}_{3} \mathrm{C}_{2} \mathrm{~T}_{x}$ flake. As an illustration, Figure 2-b provide the total atomic DOS of the $\mathrm{Ti}_{s}$ atoms calculated for $\mathrm{Ti}_{3} \mathrm{C}_{2} \mathrm{~F}_{2}$, $\mathrm{Ti}_{3} \mathrm{C}_{2} \mathrm{O}_{2}$ and the different $\mathrm{O} / \mathrm{F}$ ratios as described in the VCA: modifying $\mathrm{T}$ has a strong impact on the conduction band of the MXene with a significant shift of the first peak above $\mathrm{E}_{F}$ from 0.8 to $1.3 \mathrm{eV}$, together with a significant decrease of intensity (see the blue arrow). The same evolutions are observed on the total DOS. This peak plays a major role on the MXene optical properties since it has been shown to be involved in an interband transition drastically affecting the optical conductivity of $\mathrm{Ti}_{3} \mathrm{C}_{2}$ in the visible range. ${ }^{43}$ Figure 2-c illustrates how the total DOS at the Fermi level is also significantly modified due to changes in the surface functionalization chemistry: it is increased by a factor of approximately 2 when going from $\mathrm{Ti}_{3} \mathrm{C}_{2} \mathrm{O}_{2}$ to $\mathrm{Ti}_{3} \mathrm{C}_{2} \mathrm{~F}_{2}$, an indication for a significant change in the charge carrier density of the MXene layer. These results clearly highlight the importance of being able to identify modifications in the $\mathrm{Ti}_{s^{-}} d$ bands on a local scale using the $\mathrm{C}-\mathrm{K}$ edge as a marker.

In addition to tailoring the T-groups composition on a MXene surface, the selective desorption of surface groups, e.g., using thermal annealing in different conditions or exposure to reducing gases, to modify the MXene electronic properties, surface reactivity or graft new functional groups is also a very active research field. ${ }^{23,35,52}$ Here again, simulations show that the fine structure at the $\mathrm{C}-\mathrm{K}$ edge is a very relevant probe, evidencing sensitivity to surface vacancies through significant changes in the intensity of peak A, as shown in Figure 3-a for $\mathrm{Ti}_{3} \mathrm{C}_{2} \mathrm{O}_{x}$. Very similar results are obtained for $\mathrm{Ti}_{3} \mathrm{C}_{2} \mathrm{~F}_{x} \cdot{ }^{31}$ One can also notice that almost no change is observed in the $\mathrm{C}-\mathrm{K}$ edge fine structure at higher energy, confirming peak A as a clear marker of surface modifications, while the remaining part of the spectrum is characteristic of the inner electronic structure of the MX sheets. From a general point of view, the F-K and O-K edges are paradoxically not more sensitive to surface disorder than the $\mathrm{C}-\mathrm{K}$ edge is. This is illustrated in Figure 3-b, where the O-K edges calculated for the very same structural models as those used in Figure 3-a show that O-vacancies only noticeably affect the O-K edge for a high amount of vacancies, $75 \%$ in our simulations, similar trends being 

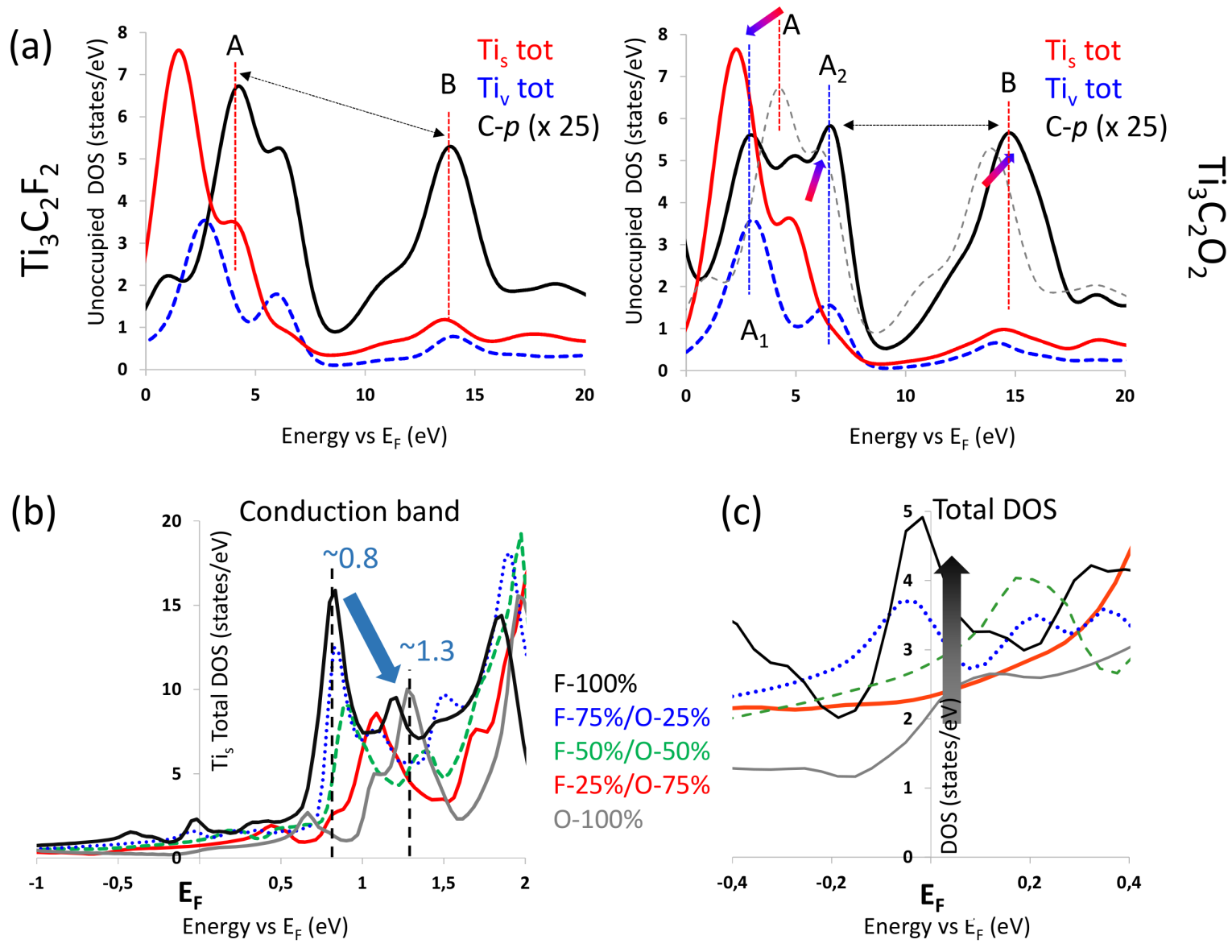

Figure 2: (a) Comparison between the total densities of states (DOS) of the surface titanium ( $\mathrm{Ti}_{s}$ - red line), volume titanium $\left(\mathrm{Ti}_{v}\right.$ - blue dashed line) and carbon atom- $p$ (black line) DOS in the $20 \mathrm{eV}$ just above the Fermi energy $\left(\mathrm{E}_{F}\right)$ for (left) $\mathrm{Ti}_{3} \mathrm{C}_{2} \mathrm{~F}_{2}$ and (right) $\mathrm{Ti}_{3} \mathrm{C}_{2} \mathrm{O}_{2}$. On the right figure, the C- $p$ DOS calculated for $\mathrm{Ti}_{3} \mathrm{C}_{2} \mathrm{~F}_{2}$ have been added for comparison (grey thin dashed line). Features corresponding to the peaks $\mathrm{A}, \mathrm{A}_{1}, \mathrm{~A}_{2}$ and $\mathrm{B}$ of figure 1-c are identified. The C- $p$ DOS is multiplied by a factor of 25 to be plotted on the same scale as the Ti DOS and a $0.7 \mathrm{eV}$ gaussian broadening was applied to the DOS in order to mimic the experimental resolution. (b) $\mathrm{Ti}_{s}$ DOS for an energy range of $3 \mathrm{eV}$ around the Fermi level for $\mathrm{Ti}_{3} \mathrm{C}_{2} \mathrm{~F}_{2}$ (black line), $\mathrm{Ti}_{3} \mathrm{C}_{2} \mathrm{~F}_{1.5} \mathrm{O}_{0.5}$ (Blue dots), $\mathrm{Ti}_{3} \mathrm{C}_{2} \mathrm{FO}$ (green dashed line), $\mathrm{Ti}_{3} \mathrm{C}_{2} \mathrm{~F}_{0.5} \mathrm{O}_{1.5}$ (red line) and $\mathrm{Ti}_{3} \mathrm{C}_{2} \mathrm{O}_{2}$ (grey line). (c) Total DOS from -0.4 to $0.4 \mathrm{eV}$ around the Fermi level. Energies are taken with respect to $\mathrm{E}_{F}$. 
obtained at the F-K edge. This is in complete agreement with experimental data showing no major evolution of the fine structure at the F-K edge upon desorption induced by in situ TEM annealing. ${ }^{35}$ Very similarly, the $\mathrm{F} / \mathrm{O}$ mixing has a rather small effect on the O-K and F-K fine structure as shown in Figure 3-c and d respectively, where two kinds of mixtures described with supercells) are considered: $25 \% \mathrm{~F} / 75 \% \mathrm{O}$ and $25 \% \mathrm{O} / 75 \% \mathrm{~F}$. The calculations at the O-K and F-K edges lead to similar fine structure, very close to what is obtained for the corresponding ideal system in both cases (i.e. $\mathrm{Ti}_{3} \mathrm{C}_{2} \mathrm{O}_{2}$ or $\mathrm{Ti}_{3} \mathrm{C}_{2} \mathrm{~F}_{2}$ respectively - black lines), and in very good agreement with experimental data (grey lines). A slight shift of the peak labeled $\mathrm{C}$ in the $\mathrm{F}-\mathrm{K}$ edge is visible, which is attributed to core-hole effects as described in supporting information-section 3.

Beyond determining the role of the surface functionalization disorder on the electronic structure of the $\mathrm{TiC}$ core in $\mathrm{Ti}_{3} \mathrm{C}_{2} \mathrm{~T}_{x}$, the $\mathrm{C}$-K edge can also be used to understand the effect of extrinsic surface perturbations like, e.g. surface impurity (like the Ca-rich impurity discussed below) or intercalated molecules resulting from the etching process. The information present in the $\mathrm{C}-\mathrm{K}$ edge can also largely be extended to the characterization of MXene sheets used as substrates in composites designed for diverse applications, ${ }^{31}$ when investigating MXenes for sensing applications or when focusing on the intercalation processes. In the former case, the interaction between the MXene and the deposited nanomaterials highlights the intended synergistic effect at the heart of the optimization strategy in designing composites, ${ }^{31,53}$ and thus appears as a key issue. As an illustration of the way the interaction between nanomaterials and MXene sheets can be evidenced on the nanoscale, Figure 4 presents the electronic structure perturbation induced in a few layers $\mathrm{Ti}_{3} \mathrm{C}_{2} \mathrm{~T}_{x}$ MXene ( $\mathrm{LiF} / \mathrm{HCl}$ etchant, see ref. ${ }^{27}$ for the synthesis protocol) by a calcium-rich impurity. In this sample, a spectrum image (SI) has been recorded in the orange marked area of Figure 4-a. The SI, given in Figure 4-b clearly highlights a brighter area, identified by a $\sim 15 \mathrm{~nm}$ side grey square, which evidences the presence of a Ca-rich impurity on or inside the MXene multilayer at this location, as revealed by the presence of the $\mathrm{Ca}-\mathrm{L}_{2,3}$ edge in the grey spec- 


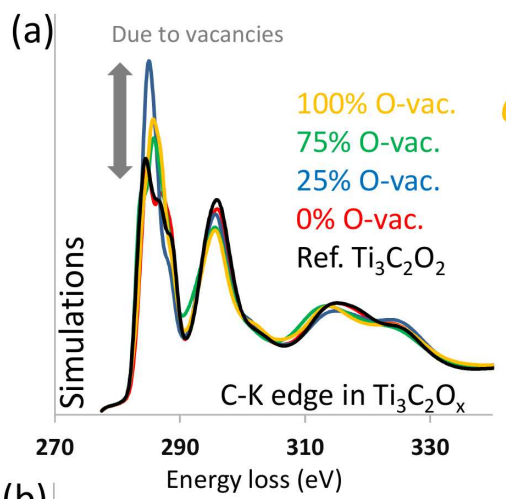

(b)

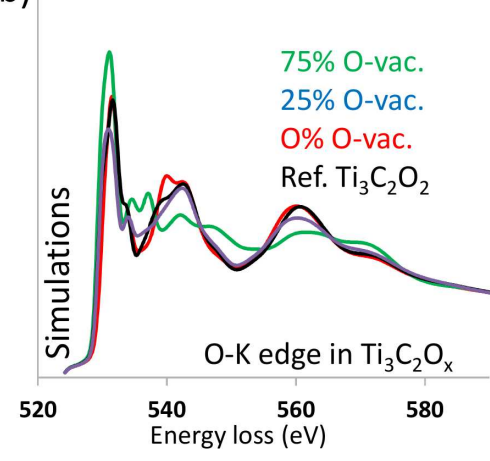

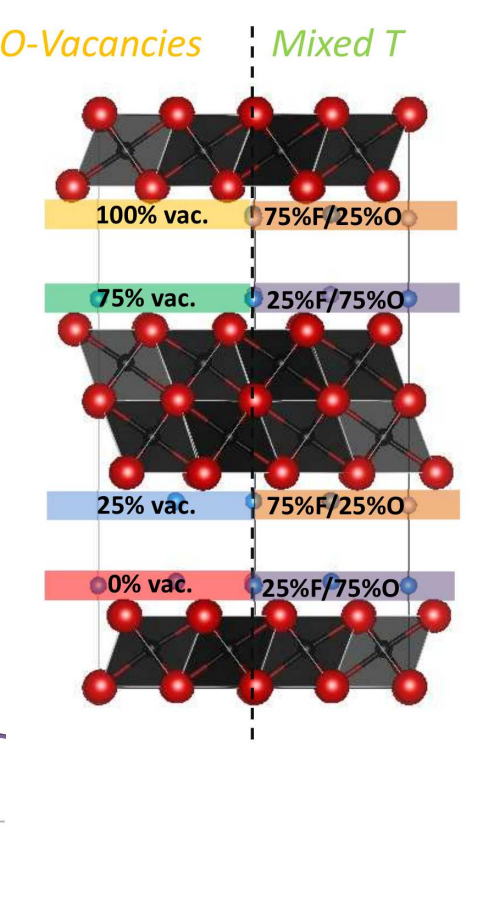

Figure 3: (a) Simulations of the C-K edges of different carbon atoms in a $\mathrm{Ti}_{3} \mathrm{C}_{2} \mathrm{O}_{x}$ multilayer having different oxygen vacancy contents on each surface. Results correspond to $\mathrm{C}$ atoms just below surfaces with $100 \%$ (orange), $75 \%$ (green), $25 \%$ (blue), $0 \%$ (red) oxygen vacancy (see the structural model in the center). The black curve corresponds to the reference spectrum calculated on a $\mathrm{Ti}_{3} \mathrm{C}_{2} \mathrm{O}_{2}$ multilayer. (b) Equivalent simulations performed at the O-K edge on the same structural model as in a). (c) Simulations of the O-K edges for oxygen atoms on the different surfaces in a $\mathrm{Ti}_{3} \mathrm{C}_{2}\left(\mathrm{O}_{x}, \mathrm{~F}_{y}\right)_{2}$ multilayer built on sheets having one $25 \% \mathrm{~F} / 75 \% \mathrm{O}$ surface (purple) and another $75 \% \mathrm{~F} / 25 \% \mathrm{O}$ (orange) (see the structural model in the center). The O-K edges obtained for each one of these surfaces are compared to the reference spectrum obtained for a $\mathrm{Ti}_{3} \mathrm{C}_{2} \mathrm{O}_{2}$ system and a typical experimental spectrum obtained on an HF-exfoliated sample. ${ }^{22}$ The experimental spectrum is vertically shifted for sake of clarity. (d) Equivalent to (c) but considering the F-K edge. 
trum in Figure 4-c. The corresponding Annular Dark Field micrograph (given in the bottom part of Figure 4-b) does not evidence such a contrast because of the similar atomic number between $\mathrm{Ca}$ and $\mathrm{Ti}$. Focusing on the $\mathrm{C}-\mathrm{K}$ edge recorded at the position of the Ca-rich impurity, the peak A intensity drops $15 \%$ (see the grey arrow) as compared to the average C-K edge obtained over the entire SI (blue curve) or to a spectrum collected on a clean part of the sheet (green square). Very importantly, the fine structure at higher energy (labelled B, $\mathrm{C}$ and $\mathrm{D})$ is not sensitive at all to this surface perturbation. Beyond the confirmation that the first peak at the $\mathrm{C}-\mathrm{K}$ edge is a very sensitive marker of the surface induced electronic structure modifications of the MXene sheets, these data show that the electronic structure of a MXene layer can be modified by the interaction with nanomaterials. In the present case, the interaction between the Ca-rich impurity (probably $\mathrm{CaF}_{x}$ since the $\mathrm{F}$ content increases on the impurity whereas the O-content remains constant) and the MXene sheet is strong enough to perturb the $\mathrm{C}-\mathrm{p} / \mathrm{Ti}_{s^{-}} d$ hybridizations discussed previously.
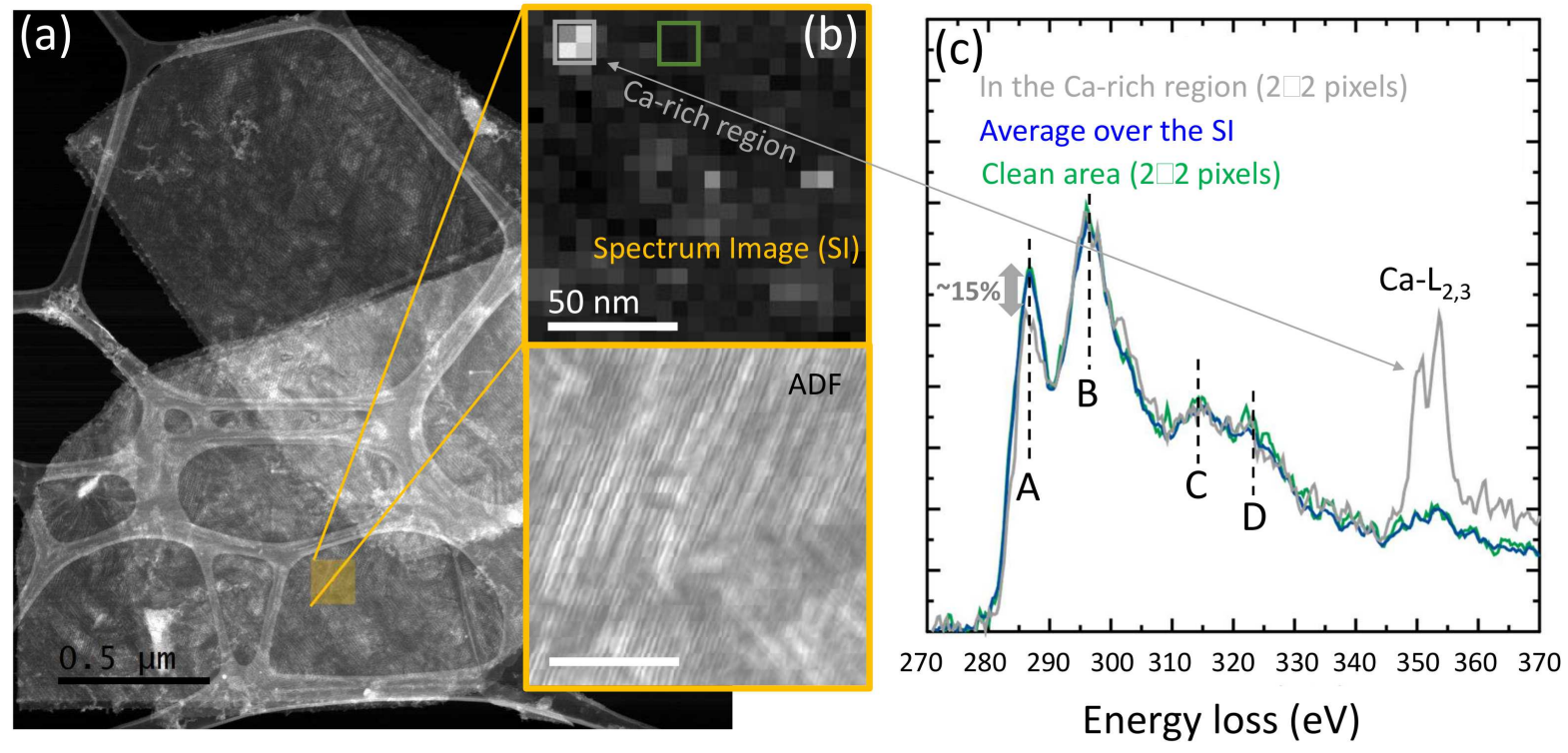

Figure 4: (a) STEM-ADF micrograph of the investigated ML- $\mathrm{Ti}_{3} \mathrm{C}_{2} \mathrm{~T}_{x}$ stack obtained using a $300 \mathrm{kV}$ electron beam. (b) Top: Spectrum image (SI) recorded on the orange area marked in (a) and integrated over the energy-range 120-730 eV, indicating a Ca-rich impurity (in grey) on the MXene. Bottom: corresponding STEM-ADF micrograph, where Moiré fringes are visible. (c) $\mathrm{C}-\mathrm{K}$ edges extracted from the SI in the Ca-rich region (grey), on a clean area (green) and compared with the average obtained over the entire SI (blue). 
The extent to which such a 15\% intensity drop of peak A reflects on the modification of the electronic structure is challenging to quantify. For this objective, we focus on the variations at the $\mathrm{C}-\mathrm{K}$ edge observed in $\mathrm{ML}-\mathrm{Ti}_{3} \mathrm{C}_{2} \mathrm{~T}_{x}$ synthesized using different etching conditions, the etchant being known to affect the density of defects in MXene sheets. ${ }^{27,32}$ We compared the electronic structure of a ML-MXene exfoliated using an anhydrous HF-pyridine etchant (see supporting information-section 1 for the description of the synthesis protocol for this sample) to three reference $\mathrm{ML}-\mathrm{Ti}_{3} \mathrm{C}_{2} \mathrm{~T}_{x}$ obtained with the $\mathrm{HF}-48 \%, \mathrm{LiF}-\mathrm{HCl}$, and $\mathrm{FeF}_{3} / \mathrm{HCl}$ etching processes (see reference ${ }^{26}$ for the synthesis protocols). The corresponding experimental data are given in Figure 5-a. Focusing first on the $\mathrm{HF}, \mathrm{LiF}-\mathrm{HCl}$, and $\mathrm{FeF}_{3} / \mathrm{HCl}$ samples, one observes that they are very similar, in agreement with previous spectra reported for the same etching processes. ${ }^{26}$ Small variations of the peak A intensity are visible: they can be attributed to the variability of surface coverage of the MXene flakes as a function of the etching process. The comparison clearly highlights the marked difference with the spectrum collected in the HF-pyridine sample where the investigated flakes were highly damaged. The fine structure is modified over the entire $\mathrm{C}-\mathrm{K}$ edge with a $18 \%$ decrease of peak A intensity, a significant broadening of structure $\mathrm{B} / \mathrm{B}^{\prime}$ and a significant intensity loss in peaks $\mathrm{C}$ and D. The fact that the entire $\mathrm{C}-\mathrm{K}$ edge is modified reveals a very significant alteration of the $\mathrm{Ti}_{3} \mathrm{C}_{2}$ skeleton in these flakes. This is confirmed in the low-loss spectra given in Figure 5-b, which reflect the electronic excitations of the valence electron gas: the peak characteristic of the $\mathrm{TiC}$ octahedra (grey dashed line) is significantly reduced in the HF-pyridine sample as compared to the other samples, confirming the degradation of the TiC octaedra. Considering the HF-pyridine sample, a very significant alteration of the TiC-core is thus associated to 18 $\%$ drop of the peak A intensity, this drop being very similar to that induced by the Ca-rich impurity on the MXene surface (i.e. $15 \%$ - see Figure 4). In conclusion, these results provide a direct evidence that the electronic structure modifications induced by the interaction of the Ca-rich impurity with the MXene surface has a significant impact on the MXene flake electronic structure and related properties. Finally, one can observe a gradual increase of the 
broadening of the structures $\mathrm{B} / \mathrm{B}^{\prime}, \mathrm{C}$ and $\mathrm{D}$ with the harshness of the etching protocol, i.e. when going from the least agressive $\mathrm{LiF} / \mathrm{HCl}$ to $\mathrm{HF}$, and finally the harshest $\mathrm{HF}$-pyridine. We thus propose the broadening of the high energy part of the $\mathrm{C}-\mathrm{K}$ edge as a qualitative marker of the density of defects induced in the MXene sheet core by different etchants, in agreement with a recent report. ${ }^{27}$ It is also quite remarkable to observe that the width of the peak $\mathrm{A}$ is almost unchanged.
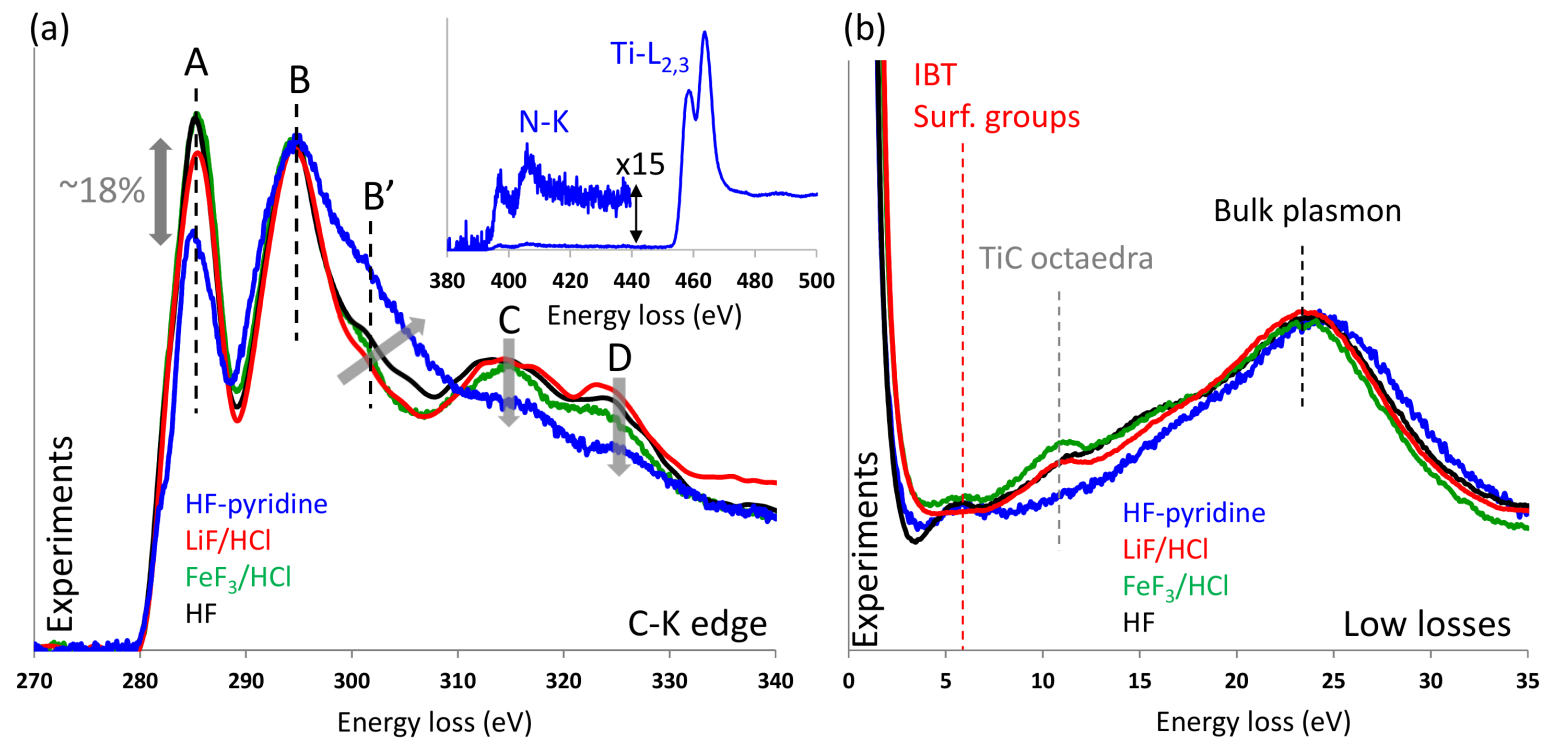

Figure 5: (a) Comparison between the C-K edges recorded in a damaged $\mathrm{ML}-\mathrm{Ti}_{3} \mathrm{C}_{2} \mathrm{~T}_{x}$ exfoliated using $\mathrm{HF}$-pyridine (blue line) and three reference spectra taken from samples obtained by the exfoliation of $\mathrm{Ti}_{3} \mathrm{AlC}_{2}$ using, $\mathrm{HF}$ (black line), $\mathrm{LiF} / \mathrm{HCl}$ (red line) and $\mathrm{FeF}_{3} / \mathrm{HCl}$ (green line). Spectra were recorded in similar conditions, at $200 \mathrm{kV}$. Inset: N-K edge recorded in the HF-pyridine sample and evidencing the presence of nitrogen within the MXene-ML. (b) Corresponding low-loss spectra. The peaks corresponding to the main characteristic excitations of the valence electron gas are highlighted: an interband transition characteristic of surface groups (red dashed line), ${ }^{43}$ a signature characteristic of the $\mathrm{TiC}$ octahedra layers (grey dashed line), and the bulk plasmon (black dashed line).

The spectrum given in the inset of Figure 5-a shows the presence of nitrogen in the HF-pyridine sample, which most probably reveals the intercalation of pyridine molecules in between the degraded MXene sheets. The intensity drop of peak A can thus also be exacerbated by inserted molecules interacting with the surface of the sheets. These measurements show how, depending on the etching process, the intercalation of molecules can significantly 
alter the MXene electronic properties. This finding is in line with the data published by Halim et al., ${ }^{9}$ where the authors showed that $\mathrm{NH}_{4}^{+} / \mathrm{NH}_{3}$ intercalated in $\mathrm{Ti}_{3} \mathrm{C}_{2} \mathrm{~T}_{x}$ thin films most likely interact with the MXene surface.

One key feature of MXenes materials is the possibility to change their elementary thickness by changing the MAX phase precursor. The thinnest sheet, $\mathrm{Ti}_{2} \mathrm{CT}_{x}$, is expected to be more sensitive to surface perturbations than $\mathrm{Ti}_{3} \mathrm{C}_{2} \mathrm{~T}_{x}$, since all titanium atoms are directly bonded to surface groups (see the structural model given in Figure 6-a). This prospect is confirmed by the experimental results given in Figure 6, where the electronic structure of a $\mathrm{ML}_{-} \mathrm{Ti}_{2} \mathrm{CT}_{x}$ flake, shown in the micrograph in Figure 6-b, has been investigated on two different areas focusing on the low energy-losses, as well as the O-K and C-K edges. Working at the single flake level allows minimizing artifacts regarding, e.g., sample aging or data processing such as deconvoluting thickness effects in the EEL spectra. The two zones of interest in Figure 6-b are identified by the blue and orange circles and labeled 1 and 2 respectively. In the low energy-loss spectra given in c, which are characteristic of the valence electron gas excitations, no difference can be seen between the two areas: the spectra are superimposable, a situation markedly different from that reported in Figure 5-b. Following the analysis given previously for $\mathrm{Ti}_{3} \mathrm{C}_{2} \mathrm{~T}_{x}$, one can conclude that the $\mathrm{TiC}$ skeleton quality of the $\mathrm{Ti}_{2} \mathrm{CT}_{x}$ layers is very similar in both areas, although probably not perfect as evidenced by the inhomogeneous contrasts and small dark regions observed in the micrograph in Figure 6-b. Contrary to the similarity of low energy-loss spectra, significant changes at the O-K edge (see Figure 6-d) reveal inhomogeneities on the $\mathrm{Ti}_{2} \mathrm{C}$ surface between the two zones: one can observe changes in the main peak A intensity together with a $1 \mathrm{eV}$ energy shift as emphasized in the inset. Although the detailed evaluation of the surface structure of this material is beyond the scope of this study, such an intensity variation linked to an energy shift is in line with the evolutions reported in Figure 6-e, which shows the O-K edges simulated for $\mathrm{Ti}_{2} \mathrm{CO}_{2}$ and $\mathrm{Ti}_{2} \mathrm{C}(\mathrm{OH})_{2}$ single layers: the intensity of peak A decreases and the maximum shifts to higher energy when going from $\mathrm{T}=\mathrm{O}$ to $\mathrm{T}=\mathrm{OH}$. Since the experimental spectra reflect a situation 
averaged between the two ideal simulated structures, the fact that the evolution observed experimentally is less pronounced than the one computed is reasonable.

(a)

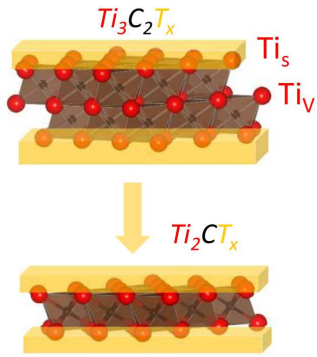

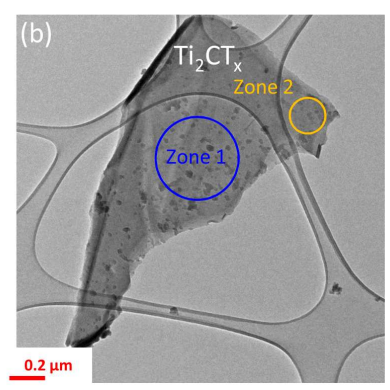

(e) (d)

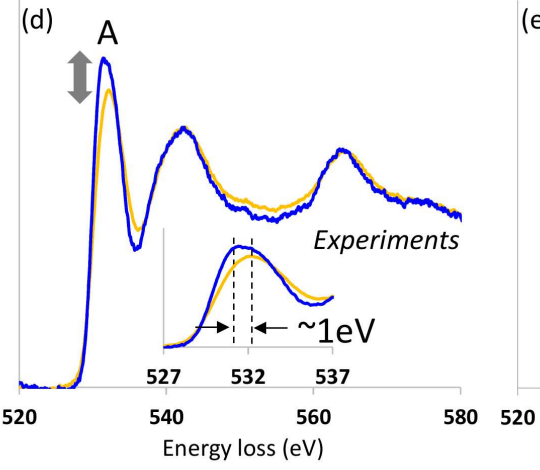

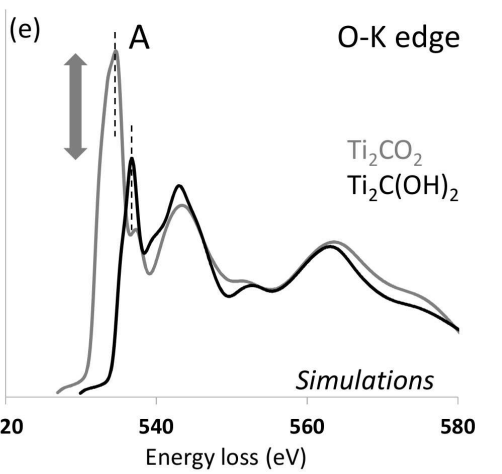
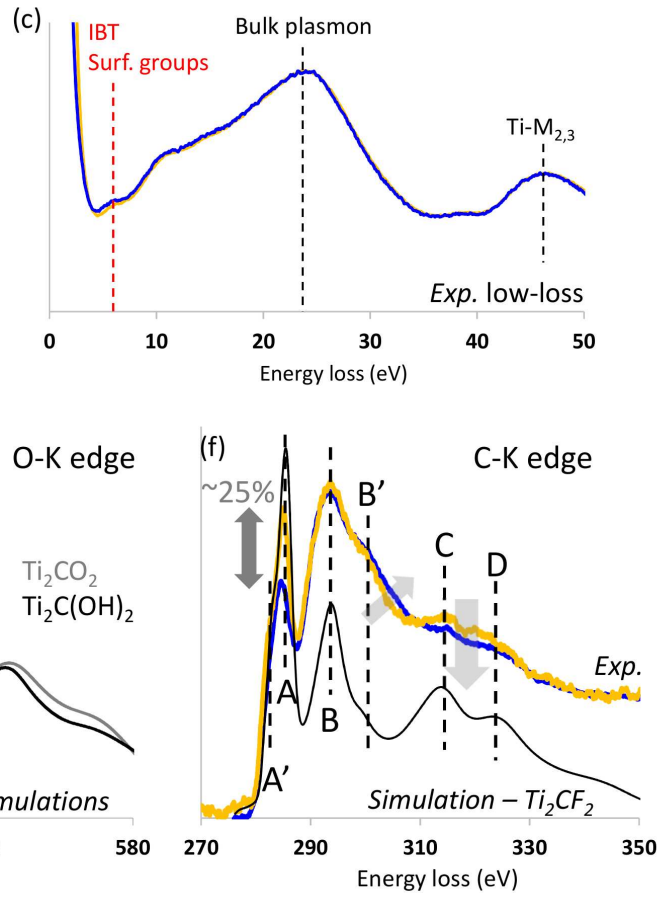

Figure 6: (a) Comparison between the structure of a $\mathrm{Ti}_{3} \mathrm{C}_{2} \mathrm{~T}_{x}$ and $\mathrm{Ti}_{2} \mathrm{CT}_{x}$ single layer. (b) TEM micrograph of the investigated $\mathrm{ML}_{-} \mathrm{Ti}_{2} \mathrm{CT}_{x}$ flake obtained from the exfoliation of $\mathrm{Ti}_{2} \mathrm{AlC}$ using the $\mathrm{FeF}_{3} / \mathrm{HCl}$ etchant: ${ }^{26}$ the zones 1 and 2 on which the different spectra given in (c), (d) and (f) were collected are highlighted in blue and orange, respectively. (c) Lowloss spectra, (d) O-K edges and (f) $\mathrm{C}-\mathrm{K}$ edges collected on zones 1 (blue) and 2 (orange). In (f), a simulation obtained on a $\mathrm{Ti}_{2} \mathrm{CF}_{2}$ single layer (black line) is also given. (e) Simulation of the O-K edge for a single $\mathrm{Ti}_{2} \mathrm{CT}_{2}$ layer (see the structural models in (a)) functionalized with $\mathrm{T}=\mathrm{O}$ (grey) or $\mathrm{OH}$ (black).

In agreement with the changes observed at the O-K edge, large changes at the C-K edges are evidenced in fig. 6-f. The intensity of peak A drops about $25 \%$ when going from zone 2 to zone 1 , which can be seen as a major difference when compared to the $15 \%$ intensity drop observed at the edge onset in $\mathrm{Ti}_{3} \mathrm{C}_{2} \mathrm{~T}_{x}$ (see Figure 4), and discussed previously. In addition, contrary to the evolution reported in Figure 4-c, where only structure A is modified, the entire spectrum changes in $\mathrm{Ti}_{2} \mathrm{CT}_{x}$ with, for instance, an intensity drop of structures $\mathrm{C}$ and D. We interpret these changes as evidences for the high sensitivity of the $\mathrm{Ti}_{2} \mathrm{C}$ layers to surface inhomogeneities as compared to $\mathrm{Ti}_{3} \mathrm{C}_{2} \mathrm{~T}_{x}$. This is confirmed by the simulations of 
the $\mathrm{C}-\mathrm{K}$ edges performed for different $\mathrm{T}$ in $\mathrm{Ti}_{2} \mathrm{CT}_{2}$, given in supporting information-section 4, which highlight the strong sensitivity of the whole fine structure at this edge to the nature of $\mathrm{T}$.

The simulation of the $\mathrm{C}-\mathrm{K}$ edge in the $\mathrm{Ti}_{2} \mathrm{CF}_{2}$ system is reported for comparison to the experiment in figure 6-f (it is similar to that obtained for $\mathrm{Ti}_{2} \mathrm{C}(\mathrm{OH})_{2}$, see supporting information section 4 for the simulations corresponding to the other compositions). The overall agreement with the experiment is rather good since all the peaks from A' to D are reproduced at the same energy positions when compared to the experiment. However, clear differences in terms of intensity ratios can be identified between the simulation and the experiments. First, the intensity of peak A, as compared to the other peaks, is much more important in the simulation. This is probably due to a combination of different factors including: (i) the fact that considering ideal functionalization, i.e. $\mathrm{F}_{2}$ in the present case, is a crude approximation to the real situation where $\mathrm{Ti}_{2} \mathrm{C}$ sheets are functionalized with a mixture of $\mathrm{F}, \mathrm{OH}$ and $\mathrm{O}$. (ii) The intensity of peak $\mathrm{A}$ in the experimental spectra is very sensitive to all kinds of disorder in the MXene multilayer as evidenced by the comparison between the C-K edges collected in zones 1 and 2: since the simulations are performed on ideal systems, it is not surprising to see such an intensity overestimation in the simulation. Concerning the peaks B to D, they are broader and less resolved in the experiments as compared to the calculation; this is quite different from the situation observed in $\mathrm{Ti}_{3} \mathrm{C}_{2} \mathrm{~T}_{2}$ (see figure 1) and at the O-K edge (see figures $6 \mathrm{~d}$ and e). This broadening is again consistent with the observed sensitivity of the $\mathrm{C}-\mathrm{K}$ edge to disorder in $\mathrm{Ti}_{2} \mathrm{C}$ (which is not considered in the simulations) and is in line with our general conclusions concerning the sensitivity to disorder of the MX core in the $\mathrm{Ti}_{2} \mathrm{C}$ system.

In terms of applications, although surface-induced modifications of the MX electronic structure could be detrimental when using MXenes as conductive supports in composites, maximizing the sensitivity of the conducting $\mathrm{TiC}$ layer to surface events is key to sensing applications. ${ }^{10}$ In this context, the higher variability at the $\mathrm{C}-\mathrm{K}$ edge in $\mathrm{Ti}_{2} \mathrm{C}$ as compared 
to $\mathrm{Ti}_{3} \mathrm{C}_{2}$ due to surface inhomogeneities, can be interpreted as an evidence for the fact that the $\mathrm{TiC}$ conducting sheet is more sensitive to surface perturbations in $\mathrm{Ti}_{2} \mathrm{C}$ than in $\mathrm{Ti}_{3} \mathrm{C}_{2}$. This is consistent with the fact that all the titanium atoms are on the surface of the MXene layer in $\mathrm{Ti}_{2} \mathrm{C}$. In that respect, these results highlight the MXene's elementary thickness as an interesting lever to consider towards the optimization of these materials for sensing applications.

\section{Conclusion}

In summary, we have shown by a detailed analysis of the role of surface disorder (i.e., Tgroups mixing, vacancies) on the C-K, O-K and F-K edge fine structure using first-principle calculations, that the C-K edge can be used as a very clear marker of surface induced electronic structure perturbations within the $\mathrm{Ti}_{3} \mathrm{C}_{2}$ conducting core of MXene sheets. The reason is that the C-K edge reflects hybridizations between the C- $p$ states and surface or volume titanium $d$ states, and thus allows discriminating between surface and core disorder on the nanometer scale. This result is very challenging to obtain by other techniques such as incoherent imaging or diffraction due to the difference in atomic numbers between the M and $\mathrm{T}$ elements. The here-provided analysis offers an approach for the clear determination of MXenes' surface disorder.

The relevance of these results is demonstrated by showing evidence of the interaction between a Ca-rich nanometer-scale impurity and a $\mathrm{ML}-\mathrm{Ti}_{3} \mathrm{C}_{2} \mathrm{~T}_{x}$, with significant effects on the MXene electronic structure. In this context, we propose the magnitude of variations of the peak A intensity at the $\mathrm{C}-\mathrm{K}$ edge onset as a probe of the strength of the interaction between a nanomaterial and its MXene support. We expect this result to be of interest in the definition of the interaction between MXene supports and deposited nanomaterials in composites. Given that the origin of peak $\mathrm{A}$ is due to hybridizations with the Ti- $d$ bands which drive the electronic properties of Ti-based MXenes, significant variations at this peak 
imply modifications of the $\mathrm{Ti}_{3} \mathrm{C}_{2}$ layer electronic structure and related properties. This result is illustrated in the case of $\mathrm{Ti}_{3} \mathrm{C}_{2}(\mathrm{O} / \mathrm{F})$ solid solutions where the reduction of the peak $\mathrm{A}$ intensity when going from $\mathrm{Ti}_{3} \mathrm{C}_{2} \mathrm{~F}_{2}$ to $\mathrm{Ti}_{3} \mathrm{C}_{2} \mathrm{O}_{2}$ goes together with a decrease of the DOS at $\mathrm{E}_{F}$ by a factor of two. This can be analyzed as a decrease of the charge carriers density by a similar amount. However, generalizing this correlation between the intensity of the peak A and the DOS around $\mathrm{E}_{F}$ to other situations (e.g. other types of surface configurations) calls for further investigations. In a complementary way, we also point out how it is possible to classify the $\mathrm{C}-\mathrm{K}$ edge fine structure recorded on different samples in terms of structural disorder by comparing the broadening of the fine structure, and correlate the density of defects to variable harshness of the etchant in the present context.

Finally, the role of the MXene layer elementary thickness on the surface sensitivity of the materials is discussed by comparing $\mathrm{Ti}_{3} \mathrm{C}_{2} \mathrm{~T}_{x}$ with $\mathrm{Ti}_{2} \mathrm{CT}_{x}$ : in the latter, an increased variability of the $\mathrm{C}-\mathrm{K}$ edge at the multilayer flake scale is observed as compared to the former. We interpret this fact as characteristic of the greater sensitivity of the electronic structure of the $\mathrm{TiC}$ core to defects and/or inhomogeneities in $\mathrm{Ti}_{2} \mathrm{CT}_{x}$. This last point illustrates the high interest that the scientific community can find in the large MXene family, where the interplay between chemistry and structure can be optimized when looking for targeted applications.

\section{Acknowledgement}

The authors acknowledge financial support from the "Région Nouvelle Aquitaine" for the PhD thesis of T. Bilyk, and the "Région Nouvelle Aquitaine" together with the European Regional Development Fund (ERDF 2014-2020) within the NanoTrans project No. PC-20162916910 for the post doctoral position of H. Pazniak. The "Agence National de la Recherche" (reference $A N R-18-C E 08-014$ - MXENECAT project) and the French research ministry (Ph.D. thesis of M. Benchakar) are acknowledged. Computations have been performed 
on the supercomputer facilities of the "Mésocentre de calcul de Poitou Charentes". The EELS experiments in TEM mode were performed at the Institut Pprime. The STEM-EELS experiments were performed at the consortium Lyon-St-Etienne de microscopie (CLYM).

\section{Supporting Information Available}

The following files are available free of charge.

Synthesis protocol and characterization of the $\mathrm{Ti}_{3} \mathrm{C}_{2} \mathrm{~T}_{x}$ samples obtained with the HFpyridine etchant (Figure S1), discussion on the validity of the virtual crystal approximation for the description of $\mathrm{F} / \mathrm{O}$ disorder in $\mathrm{Ti}_{3} \mathrm{C}_{2} \mathrm{OF}$ with a comparison with a supercell-based approach (Figure S2 and S3), analysis of the core-hole screening at the O and F-edges in $\mathrm{Ti}_{3} \mathrm{C}_{2} \mathrm{O}_{2}$ and $\mathrm{Ti}_{3} \mathrm{C}_{2} \mathrm{~F}_{2}$ (Figure S4), simulations of the C-K and F-K edges in $\mathrm{Ti}_{2} \mathrm{CT}_{2}$ single layer (Figure S4).

This material is available free of charge via the Internet at http://pubs.acs.org/.

\section{References}

(1) Naguib, M.; Kurtoglu, M.; Presser, V.; Lu, J.; Niu, J.; Heon, M.; Hultman, L.; Gogotsi, Y.; Barsoum, M. W. Two-Dimensional Nanocrystals Produced by Exfoliation of $\mathrm{Ti}_{3} \mathrm{AlC}_{2}$. Adv. Mater. 2011, 23, 4248-4253.

(2) Naguib, M.; Mashtalir, O.; Carle, J.; Presser, V.; Lu, J.; Hultman, L.; Gogotsi, Y.; Barsoum, M. W. Two-Dimensional Transition Metal Carbides. ACS Nano 2012, 6, 1322-1331.

(3) Ghidiu, M.; Lukatskaya, M. R.; Zhao, M.-Q.; Gogotsi, Y.; Barsoum, M. W. Conductive two-dimensional titanium carbide "clay "with highvolumetric capacitance. Nature 2014, 516, 78-81. 
(4) Celerier, S.; Hurand, S.; Garnero, C.; Morisset, S.; Benchakar, M.; Habrioux, A.; Chartier, P.; Mauchamp, V.; Findling, N.; Lanson, B.; Ferrage, E. Hydration of $\mathrm{Ti}_{3} \mathrm{C}_{2} \mathrm{~T}_{x}$ MXene: An Interstratification Process with Major Implications on Physical Properties. Chem. Mater 2019, 31, 454-461.

(5) Anasori, B.; Lukatskaya, M. R.; Gogotsi, Y. 2D metal carbides and nitrides (MXenes) for energy storage. Nature Reviews Materials 2017, 2, 16098.

(6) Rasool, K.; Pandey, R. P.; Rasheed, A. P.; Buczek, S.; Gogotsi, Y.; Mahmoud, K. A. Water treatment and environmental remediation applications of twodimensional metal carbides (MXenes). Materials Today 2019, 30, 80-102.

(7) Shahzad, F.; Alhabeb, M.; Hatter, C. B.; Anasori, B.; Hong, S. M.; Koo, C. M.; Gogotsi, Y. Electromagnetic interference shielding with 2D transition metal carbides (MXenes). Science 2016, 353, 1137-1140.

(8) Hantanasirisakul, K.; Gogotsi, Y. Electronic and Optical Properties of 2D Transition Metal Carbides and Nitrides (MXenes). Adv. Mater. 2018, 30, 1804779.

(9) Halim, J.; Lukatskaya, M. R.; Cook, K. M.; Lu, J.; Smith, C.; Näslund, L.-A.; May, S. J.; Hultman, L.; Gogotsi, Y.; Eklund, P.; Barsoum, M. W. Transparent Conductive Two-Dimensional Titanium Carbide Epitaxial Thin Films. Chem. Mater. 2014, 26, 2374-2381.

(10) Kim, S. J.; Koh, H.-J.; Ren, C. E.; Kwon, O.; Maleski, K.; Cho, S.-Y.; Anasori, B.; Kim, C.-K.; Choi, Y.-K.; Kim, J.; Gogotsi, Y.; Jung, H.-T. Metallic $\mathrm{Ti}_{3} \mathrm{C}_{2} \mathrm{~T}_{x}$ MXene Gas Sensors with Ultrahigh Signal-to-Noise Ratio. ACS Nano 2018, 12, 986-993.

(11) Alhabeb, M.; Maleski, K.; Anasori, B.; Lelyukh, P.; Clark, L.; Sin, S.; Gogotsi, Y. Guidelines for Synthesis and Processing of Two-Dimensional Titanium Carbide $\left(\mathrm{Ti}_{3} \mathrm{C}_{2} \mathrm{~T}_{x}\right.$ MXene). Chem. Mater. 2017, 29, 7633-7644. 
(12) Barsoum, M. W. MAX Phases: Properties of Machinable Ternary Carbides and Nitrides, 1st ed.; Wiley, 2013.

(13) Sokol, M.; Natu, V.; Kota, S.; Barsoum, M. W. On the Chemical Diversity of the MAX Phases. Trends in Chemistry 2019, 1, 210-223.

(14) Gogotsi, Y.; Anasori, B. The rise of MXenes. ACS Nano 2019, 13, 8491-8494.

(15) Tao, Q.; Dahlqvist, M.; Lu, J.; Kota, S.; Meshkian, R.; Halim, J.; Palisaitis, J.; Hultman, L.; Barsoum, M. W.; Persson, P. O.; Rosén J. Two-dimensional $\mathrm{Mo}_{1.33} \mathrm{C}$ MXene with divacancy ordering prepared from parent 3D laminate with in-plane chemical ordering. Nature Communications 2017, 8, 14949.

(16) Anasori, B.; Shi, C.; Moon, E. J.; Xie, Y.; Voigt, C. A.; Kent, P. R.; May, S. J.; Billinge, S. J.; Barsoum, M. W.; Gogotsi, Y. Control of electronic properties of 2D carbides (MXenes) by manipulating their transition metal layers. Nanoscale Horiz. 2016, 1, 227-234.

(17) Fu, Z.; Zhang, Q.; Legut, D.; Si, C.; Germann, T.; Lookman, T.; Du, S. Y.; Franisco, J. S.; Zhang, R. F. Stabilization and strengthening effects of functional groups in two-dimensional titanium carbide. Physical Review B 2016, 94, 104103.

(18) Khazaei, M.; Ranjbar, A.; Arai, M.; Sasakib, T.; Yunoki, S. Electronic properties and applications of MXenes: a theoretical review. Journal of Materials Chemistry C 2017, 5, 2488-2503.

(19) Frey, N. C.; Bandyopadhyay, A.; Kumar, H.; Anasori, B.; Gogotsi, Y.; Shenoy, V. B. Surface-Engineered MXenes: Electric Field Control of Magnetism and Enhanced Magnetic Anisotropy. ACS Nano 2019, 13, 2831-2839. 
(20) Kamysbayev, V.; Filatov, A. S.; Hu1, H.; Rui, X.; Lagunas, F.; Wang, D.; Klie, R. F.; Talapin1, D. V. Covalent surface modifications and superconductivity of two-dimensional metal carbide MXenes. Science 2020, 369, 979-983.

(21) Halim, J.; Cook, K. M.; Naguib, M.; Eklund, P.; Gogotsi, Y.; Rosén, J.; Barsoum, M. W. X-ray photoelectron spectroscopy of select multi-layered transition metal carbides (MXenes). Applied Surface Science 2016, 362, 406-417.

(22) Magne, D.; Mauchamp, V.; Celerier, S.; Chartier, P.; Cabioc'h, T. Siteprojected electronic structure of two-dimensional $\mathrm{Ti}_{3} \mathrm{C}_{2}$ MXene: the role of the surface functionalization groups. Physical Chemistry Chemical Physics 2016, 18, 30946-30953.

(23) Persson, I.; Naslund, L.-A.; Halim, J.; Barsoum, M. W.; Darakchieva, V.; Palisaitis, J.; Rosén, J.; Persson, P.O. On the organization and thermal behavior of functional groups on $\mathrm{Ti}_{3} \mathrm{C}_{2}$ MXene surfaces in vacuum. 2D Materials 2018, $5,015002$.

(24) S hultz, T.; Frey, N. C.; Hantanasirisakul, K.; Park, S.; May, S.; Shenoy, V.; Gogotsi, Y.; Koch, N. Surface Termination Dependent Work Function and Electronic Properties of $\mathrm{Ti}_{3} \mathrm{C}_{2} \mathrm{~T}_{x}$ MXene. Chemistry of Materials 2019, 31, 6590-6597.

(25) Wang, H.-W.; Naguib, M.; Page, K.; Wesolowski, D. J.; Gogotsi, Y. Resolving the Structure of $\mathrm{Ti}_{3} \mathrm{C}_{2} \mathrm{~T}_{x}$ MXenes through Multilevel Structural Modeling of the Atomic Pair Distribution Function. Chemistry of Materials 2016, 28, 349359.

(26) Wang, X.; Garnero, C.; Rochard, G.; Magne, D.; Morisset, S.; Hurand, S.; Chartier, P.; Rousseau, J.; Cabioch, T.; Coutanceau, C.; Mauchamp, V.; 
Celerier, S. A new etching environment $\left(\mathrm{FeF}_{3} / \mathrm{HCl}\right)$ for the synthesis of twodimensional titanium carbide MXenes: a route towards selective reactivity vs. water. Journal of Materials Chemistry A 2017, 5, 22012-22023.

(27) Benchakar, M.; Loupias, L.; Garnero, C.; Bilyk, T.; Morais, C.; Canaff, C.; Guignard, N.; Morisset, S.; Pazniak, H.; Hurand, S.; et al. One MAX phase, different MXenes: a guideline to understand the crucial role of etching conditions on $\mathrm{Ti}_{3} \mathrm{C}_{2} \mathrm{~T}_{x}$ surface chemistry. Applied Surface Science $\mathbf{2 0 2 0}$, 530, 147209

(28) Lu, J.; Persson, I.; Lind, H.; Palisaitis, J.; Li, M.; Li, Y.; Chen, K.; Zhou, J.; Du, S.; Chai, Z.; Huang, Z.; Hultman, L.; Eklund, P.; Rosén, J.; Huangband Q.; Persson, P.A.O. $\mathrm{Ti}_{n+1} \mathrm{C}_{n}$ MXenes with fully saturated and thermallystable Cl terminations Nanoscale Advances 2019, 1, 3680-3685.

(29) Ming Hong Ng, V.; Huang, H.; Zhou, K.; See Lee, P.; Que, W.; Zhichuan Xu, J.; Bing Kong, L. Recent progress in layered transition metal carbides and/or nitrides (MXenes) and their composites: synthesis and applications. Journal of Materials Chemistry A 2017, 5, 3039.

(30) Liu, Y.-T.; Zhang, P.; Sun, N.; Anasori, B.; Zhu, Q.-Z.; Liu, H.; Gogotsi, Y.; Xu, B. Self-Assembly of Transition Metal Oxide Nanostructures on MXene Nanosheets for Fast and Stable Lithium Storage. Adv. Mater. 2018, 30, 1707334 .

(31) Benchakar, M.; Bilyk, T.; Garnero, C.; Loupias, L.; Morais, C.; Pacaud, J.; Canaff, C.; Chartier, P.; Morisset, S.; Guignard, N.; Mauchamp, V.; Celerier, S.; Habrioux, A. MXene Supported Cobalt Layered Double Hydroxide Nanocrystals: Facile Synthesis Route for a Synergistic Oxygen Evolution Reaction Electrocatalyst. Adv. Mater. Interf. 2019, 6, 1901328.

(32) Sang, X.; Xie, Y.; Lin, M.-W.; Alhabeb, M.; Van Aken, K. L.; Gogotsi, Y.; 
Kent, P. R.; Xiao, K.; Unocic, R. R. Atomic defects in Monolayer Titanium Carbide $\left(\mathrm{Ti}_{3} \mathrm{C}_{2} \mathrm{~T}_{x}\right)$ MXene. ACS Nano 2016, 10, 9193-9200.

(33) Sang, X.; Xie, Y.; Yilmaz, D. E.; Lotfi, R.; Alhabeb, M.; Ostadhossein, A.; Anasori, B.; Sun, W.; Li, X.; Xiao, K.; Kent, P. R.; van Duin, A. C.; Gogotsi, Y.; Unocic, R. R. In situ atomistic insight into the growth mechanisms of single layer 2D transition metal carbides. Nature Communications 2018, 9, 2266.

(34) Hart, J. L.; Hantanasirisakul, K.; Lang, A. C.; Anasori, B.; Pinto, D.; Pivak, Y.; van Omme J. Tijn,; May, S. J.; Gogotsi, Y.; Taheri, M. L. Control of MXenes'electronic properties through termination and intercalation. Nature Communications 2019, 10, 522 .

(35) El-Demellawi, J. K.; Lopatin, S.; Yin, J.; Mohammed, O. F.; Alshareef, H. N. Tunable Multipolar Surface Plasmons in $2 \mathrm{D} \mathrm{Ti}_{3} \mathrm{C}_{2} \mathrm{~T}_{x}$ MXene Flakes. ACS Nano 2018, 12, 8485-8493.

(36) Mauchamp, V.; Bugnet, M.; Bellido, E. P.; Botton, G. A.; Moreau, P.; Magne, D.; Naguib, M.; Cabioc'h, T.; Barsoum, M. W. Enhanced and tunable surface plasmons in two-dimensional $\mathrm{Ti}_{3} \mathrm{C}_{2}$ stacks: Electronic structure versus boundary effects. Physical Review B 2014, 89, 235428.

(37) Rasool, H. I.; Ophus, C.; Zettl, A. Atomic Defects in Two Dimensional Materials. Adv. Mater. 2015, 27, 5771-5777.

(38) Bottari, G.; Herranz, M. A.; Wibmer, L.; Volland, M.; Rodriguez-Perez, L.; Guldi, D. M.; Hirsch, A.; Martín, N.; D’Souza, F.; Torres, T. Chemical functionalization and characterization of graphene-based materials. Chem. Soc. Rev. 2017, 46, 4464-4500.

(39) Blaha, P.; Schwarz, K.; Madsen, G.; Kvasnicka, D.; Luitz, J.; Laskowski, R.; Tran, F.; Marks L.D. WIEN2k: An Augmented Plane Wave Plus Local Orbitals 
Program for Calculating Crystal Properties; Vienna University of Technology, Austria, 2018.

(40) Blaha, P.; Schwarz, K.; Tran, F.; Laskowski, R.; Madsen, G. K. H.; Marks, L. D. WIEN2k: An APW+lo program for calculating the properties of solids. The Journal of Chemical Physics 2020, 152, 074101.

(41) Perdew, J. P.; Burke, K.; Ernzerhof, M. Generalized Gradient Approximation Made Simple. Phys. Rev. Lett. 1996, 77, 3865-3868.

(42) Magne, D.; Mauchamp, V.; Celerier, S.; Chartier, P.; Cabioc'h, T. Spectroscopic evidence in the visible-ultraviolet energy range of surface functionalization sites in the multilayer $\mathrm{Ti}_{3} \mathrm{C}_{2}$ MXene. Physical Review $B$ 2015, 91, 201409(R).

(43) Xie, Y.; Kent, P. R. C. Hybrid density functional study of structural and electronic properties of functionalized $\mathrm{Ti}_{n+1} \mathrm{X}_{n}(\mathrm{X}=\mathrm{C}, \mathrm{N})$ monolayers. Phys. Rev. B 2013, 87, 235441.

(44) Kresse, G.; Furthmüller, J. Efficient iterative schemes for ab initio total-energy calculations using a plane-wave basis set. Phys. Rev. B 1996, 54, 11169-11186.

(45) Kresse, G.; Joubert, D. From ultrasoft pseudopotentials to the projector augmented-wave method. Phys. Rev. B 1999, 59, 1758-1775.

(46) Hebert, C. Practical aspects of running the WIEN2k code for electron spectroscopy. Micron 2007, 38, 12-28.

(47) Bugnet, M.; Mauchamp, V.; Eklund, P.; Jaouen, M.; Cabioc’h, T. Contribution of core-loss fine structures to the characterization of ion irradiation damages in the nanolaminated ceramic $\mathrm{Ti}_{3} \mathrm{AlC}_{2}$. Acta Materialia 2013, 61, 7348-7363. 
(48) Bugnet, M.; Jaouen, M.; Mauchamp, V.; Cabioc'h, T.; Hug, G. Experimental and first-principles investigation of the electronic structure anisotropy of $\mathrm{Cr}_{2}$ AlC. Physical Review B 2014, 90, 195116.

(49) Momma, F.; Izumi, F. VESTA 3 for three-dimensional visualization of crystal, volumetric and morphology data. Journal of Applied Crystallography 2011, 44, $1272-1276$.

(50) Persson, I.; Halim, J.; Lind, H.; Hansen, T. W.; Wagner, J. B.; Naslund, L.-A.; Darakchieva, V.; Palisaitis, J.; Rosén, J.; Persson, P. O. A. 2D Transition Metal Carbides (MXenes) for Carbon Capture. Adv. Mater. 2018, 31, 1805472.

(51) Lang, Z.; Zhuang, Z.; Li, S.; Xia, L.; Zhao, Y.; Zhao, Y.; Han, C.; Zhou, L. MXene Surface Terminations Enable Strong Metal-Support Interactions for Efficient Methanol Oxidation on Palladium. ACS Applied Materials and Interfaces 2020, 12, 2400. 
Graphical TOC Entry

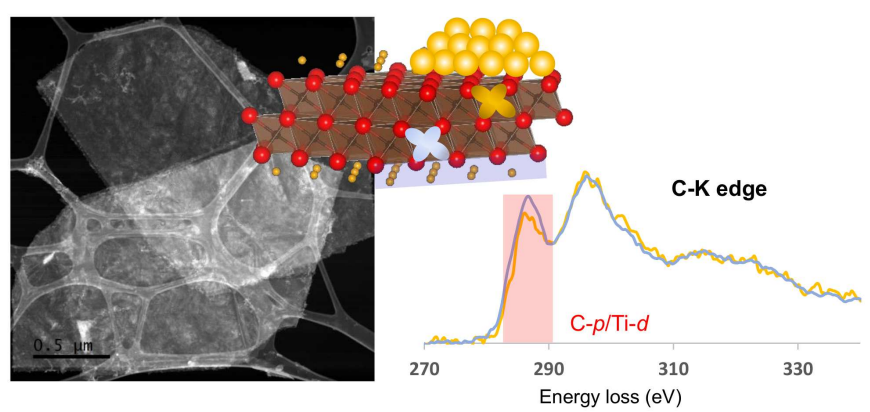

